\title{
Implementation of an inpatient smoking cessation programme in a Veterans Affairs facility
}

\author{
Lowryanne Vick, Sonia A Duffy, Lee A Ewing, Kathryn Rugen and Connie Zak
}

Aims and objectives. To test the transportability and implementation of the Tobacco Tactics intervention using the Reach, Effectiveness, Adoption, Implementation and Maintenance framework, for inpatient units at the Jesse Brown Veterans Affairs Medical Center.

Background. Smoking rates are high among veterans. While the Department of Veterans Affairs has standardised outpatient cessation clinics, inpatient cessation services, known to be efficacious, are only sporadically provided.

Design. This was a phase 4 , pre and postimplementation study of the Tobacco Tactics intervention.

Methods. A unique convenience sample of inpatient veteran smokers was recruited both before $(n=54)$ and after $(n=50)$ implementation of the Tobacco Tactics programme. Participants completed baseline and 30-day follow-up surveys along with urine cotinine test kits. In addition, staff completed anonymous surveys during the preintervention period $(n=158)$ and two months after $(n=81)$ the Tobacco Tactics training. Bivariate analyses compared preintervention vs. postintervention patient and staff characteristics using Chi-square, Fisher's Exact or Student's $t$-test. $p$-values $<0 \cdot 05$ were considered significant.

Results. Patient-reported receipt of services and satisfaction was $10 \%$ higher in the postintervention compared to the preintervention group. Quit rates were 3\% higher in the postintervention than in the preintervention group. The mean number of cigarettes smoked per day increased from 13 to 15 in the preintervention group, while the mean number of cigarettes smoked per day decreased from 14 to 9 in the postintervention group. Staff's confidence in their ability to provide cessation services improved greatly posttraining $(p=0.0017)$ as did self-reported delivery of cessation services $(p=0 \cdot 0154)$.

Conclusions. With as little as one-hour training for nurses, the Tobacco Tactics intervention has the potential to be widely disseminated in the Department of Veterans Affairs.

Relevance to clinical practice. The implementation of inpatient smoking interventions has the potential to improve quit rates and decrease morbidity and mortality in the Department of Veterans Affairs.

Key words: health services research, nursing intervention, research implementation

Accepted for publication: 26 February 2012

Authors: Lowryanne Vick, DNP, APN, ACNP-BC, Nurse Practitioner, Cardiology Service, Jesse Brown VA Medical Center, Chicago, IL; Sonia A Duffy, PhD, RN, Research Investigator, Ann Arbor VA Center for Clinical Management Research and Associate Professor, University of Michigan, School of Nursing, Departments of Otolaryngology and Psychiatry, Ann Arbor, MI; Lee A Ewing, MPH, Research Health Science Specialist, Ann Arbor VA Center for Clinical Management Research, Ann Arbor, MI; Kathryn Rugen, PhD, FNP-BC, Nurse Consultant, VA Centers of Excellence in Primary Care Education, Office of Academic Affiliations, Washington, DC, Associate Chief Nurse for Education and
Research, Jesse Brown VA Medical Center Chicago, IL and Assistant Professor, University of Illinois at Chicago, College of Nursing, Chicago, IL; Connie Zak, DNP, MBA, FNP-BC, Associate Professor, Associate Dean for Scholarship and Director of Graduate Studies, Lewis University, College of Nursing and Health Professions, Romeoville, IL, USA

Correspondence: Sonia A Duffy, Research Investigator, Ann Arbor VA Center for Clinical Management Research, 2215 Fuller Road, Ann Arbor, MI 48105, USA. Telephone: +1 7343950613.

E-mail: bump@umich.edu 


\section{Introduction}

Cigarette smoking causes approximately 438,000 deaths annually (Centers for Disease Control and Prevention 2007) and is disproportionately higher among veterans (Cui et al. 2006, Sherman et al. 2006, Hamlett-Berry et al. 2009). The efficacious, nurse-administered, Tobacco Tactics intervention was tested in the Department of Veterans Affairs (VA) among head and neck cancer patients, a group of highly recalcitrant smokers (Duffy et al. 2006). Since that randomised control trial, the Tobacco Tactics intervention was refined and packaged into a toolkit for nurses and patients and disseminated in a VA Service Directed Project (Duffy et al. 2010a). This project tested the transportability of the Tobacco Tactics intervention to the Jesse Brown VA Medical Center (JBVAMC).

\section{Background}

\section{Smoking services in the VA}

As a result of the high prevalence of tobacco use among military personnel, the Department of Defense spends an estimated \$564 million per year for smoking-related medical care (Dall et al. 2007). VA practitioners exceed national standards in terms of identifying smokers and offering general quit advice (Sherman et al. 2008). However, in the VA, smoking cessation services are offered sometimes in primary care, but primarily in the outpatient setting (Duffy et al. 2008). Unfortunately, outpatient cessation programmes tend to be underutilised among the smoking population (Miller et al. 1997). In contrast, inpatient cessation programmes have the potential to reach a vast number of smokers (Hennrikus et al. 2005, Duffy et al. 2008, Wolfenden et al. 2008).

\section{Nurse-based smoking cessation interventions}

With approximately 2.9 million registered nurses (RNs) in the United States, nurses are the largest number of front line providers (The Center for Nursing Advocacy 2006) and thus nurses interact with patients more than any other healthcare provider (Chouinard \& Robichaud-Ekstrand 2005). It is well documented that nurses effectively deliver smoking cessation interventions (Gomm et al. 2002, Thompson et al. 2007, Gies et al. 2008, Ergul \& Temel 2009, Rice \& Stead 2008, Duffy et al. 2010a). Although nursing staff is ideally situated to provide smoking cessation services, several barriers exist that prevent nurses from delivering these services, including competing priorities, lack of knowledge and training, lack of confidence in the ability to provide appropriate smoking cessation support and concern about upsetting patients (Duffy et al. 2008, Gies et al. 2008). Hence, many inpatient healthcare providers fail to seize the window of opportunity during hospitalisation to provide cessation services (Duffy et al. 2008, Ginn et al. 2008).

\section{Current evidence-based inpatient cessation interventions}

Hospitalisation creates an excellent opportunity to implement smoking cessation efforts, as hospitalised patients are in a heightened state of readiness to quit smoking (Davies et al. 2005); many tobacco users are admitted for smokingrelated illnesses making them more receptive to cessation interventions (Rigotti et al. 2007, Duffy et al. 2008, Fiore et al. 2008, Ginn et al. 2008, Wolfenden et al. 2008). Additionally, the hospital setting can increase access to smoking cessation interventions, decrease exposure to environmental smoking cues and create an environment that is unfavourable to smoking (Duffy et al. 2008, Wolfenden et al. 2008, Targhetta et al. 2011). A Cochrane review conducted by Rigotti et al. (2007) provided support for inpatient smoking cessation programmes that are multicomponent and provide cessation medications, counselling and provide at least one month of supportive contact postdischarge. The Tobacco Tactics programme is a multicomponent, inpatient smoking cessation programme that incorporates guidelines from the Agency for Healthcare Research and Quality (AHRQ) for treatment of smoking and is individualised to meet the needs of the hospitalised veteran who smokes. The Tobacco Tactics programme has been found to increase the receipt of nurse-delivered smoking cessation (Duffy et al. 2006, 2010a).

\section{Theoretical model}

Numerous theoretical models have been used to incorporate research into patient care including the Reach, Effectiveness, Adoption, Implementation and Maintenance (RE-AIM) framework used in this study (Abildso et al. 2010, Caperchione \& Coulson 2010). The overall goal of the RE-AIM framework is to enhance the applicability of a research-based intervention in clinical practice (RE-AIM.org). Utilisation of the RE-AIM framework is intended to ease the process of planning, conducting, reporting and selecting interventions to be implemented on a large scale (Kaiser Permanente Colorado Region Institute for Health Research 2010). In addition, the RE-AIM framework assists in determining the individual and global impact of a given intervention (Glasgow et al. 2006). Evaluation of smoking cessation interventions, using the RE-AIM 
framework, has been demonstrated in previous publications (France et al. 2001, Prochaska et al. 2009, Reid et al. 2010, Anesetti-Rothermel et al. 2011, Meyer et al. 2011).

The constructs of the RE-AIM framework are: (1) Reach (quantity of individuals in receipt of an intervention), (2) Effectiveness (impact of an intervention on measured outcomes), (3) Adoption (proportion of agents willing to deliver the intervention), (4) Implementation (extent to which the intervention is implemented as intended), and (5) Maintenance (long-term sustainability of an intervention) (Bakken \& Ruland 2009). Reflecting the RE-AIM framework, the specific aims of this study were to: (1) determine if inpatient smokers report an increase in receipt of services and satisfaction with services (Reach), (2) measure cessation rates after implementation of the intervention (Effectiveness), (3) identify the number of inpatient nurses who were trained to conduct the Tobacco Tactics intervention, determine the degree and quality of implementation of the intervention and assess change in staff attitudes and behaviours about the delivery of cessation services preimplementation and postimplementation of the Tobacco Tactics intervention (Adoption), (4) identify preintervention and postintervention self-reported implementation of specific components of the Tobacco Tactics intervention (Implementation), and (5) evaluate the sustainability of the intervention (Maintenance).

\section{Methods}

\section{Design}

This quasi-experimental, phase 4 implementation study used a preintervention, postintervention design to assess moving the evidence-based Tobacco Tactics programme into practice (Titler 2004). In this design, two unique convenience samples of veteran inpatient smokers were recruited before and after the hospital-wide roll-out of the Tobacco Tactics intervention and compared on self-reported receipt of and statisfaction with cessation services (Reach) and posthospitalisation quit rates (Effectiveness); the preintervention and postintervention samples were not linked. To evaluate the preintervention and postintervention changes in the number of inpatient providers trained (Adoption), self-reported implementation of the Tobacco Tactics intervention (Implementation), and sustainability of the intervention (Maintenance), two convenience samples of providers were also surveyed before and after the roll-out of the intervention; the preintervention and postintervention provider samples may have had some overlap of participants, but were not necessarily the same nurses and were not linked. Human studies approval was obtained from the JBVAMC/Northwestern University/University of Illinois at Chicago (UIC) collaborative Institutional Review Board and the JBVAMC Department of Research and Development.

\section{Setting and sample}

The setting was the JBVAMC, an urban, 200-bed acute care facility which provides services to approximately 58,000 veterans. The veterans who receive care at the JBVAMC are predominately African-American men of low socioeconomic status (SES).

To evaluate the Reach and Effectiveness of the intervention in accordance with the RE-AIM framework, this study included two unique convenience samples of veteran inpatient smokers recruited before and after the hospital-wide roll-out of the Tobacco Tactics intervention that were: (1) over the age of $18,(2)$ admitted as inpatients to general medical, surgical, intensive care unit, telemetry, psychiatric and extended care units, and (3) willing to complete the smoking survey. Exclusion criterions were those veterans who were: (1) too ill or impaired to participate, (2) in terminal stages of illness, and (3) non-English speaking. Patients were recruited both preintervention and postintervention.

To evaluate the Adoption, Implementation and Maintenance of the study in accordance with the RE-AIM framework, two convenience samples of providers were surveyed preintervention and postintervention; some, but not all of the preintervention respondents, were also included in the postintervention sample. Registered nurses who worked on the inpatient units were specifically targeted in this study. Other healthcare providers (i.e. outpatient staff nurses, respiratory therapists and licensed practical nurses) interested in smoking cessation were also included.

\section{Procedures}

\section{Patient recruitment}

Veteran smokers were identified by obtaining an electronically generated list of daily admissions and then reviewing the Computerized Patient Record System (CPRS) to identify smokers. Unique inpatient smokers were approached by a co-investigator or graduate nursing student at their bedside and, after signing informed consent, completed a baseline survey in both the preintervention and postintervention period. The preintervention group was recruited prior to the implementation of the Tobacco Tactics intervention and received usual care whereas the postintervention group was recruited following the implementation of the intervention and had the chance for exposure to the Tobacco Tactics programme. Fifty patients were targeted in both the 
preintervention and postintervention period, which was less than what was needed to be powered to detect significant differences in quit rates, but enough to determine whether results were in the expected direction for this implementation study. The response rate was $50 \%$ in the preintervention group and $38 \%$ in the postintervention group.

In addition, a co-investigator collected general information from CPRS about their admission and medical conditions. Findings from the chart review were documented on the Medical History Instrument Form. This form contained information such as the unit where the patient received the Tobacco Tactics intervention, dates of admission and discharge, admission and discharge diagnoses, comorbidities, length of stay, transfer and surgery dates (if applicable).

To evaluate differences in preintervention and postintervention receipt of and satisfaction with services (Reach) and quit rates (Effectiveness), 30-day follow-up surveys and urine cotinine tests were mailed to participants using a modified Dillman (1978) technique. All participants, regardless of their exposure to the intervention, received a follow-up survey along with a urine cotinine test kit 30 days postdischarge. Participants were given $\$ 5.00$ canteen vouchers for each survey and $\$ 15 \cdot 00$ canteen vouchers for returning the cotinine test.

\section{Staff recruitment}

To identify preintervention and postintervention staff Adoption, Implementation and Maintenance behaviours regarding the provision of smoking cessation services, staff were given an anonymous survey at the beginning of each training session. Staff attendance at the training sessions was strongly encouraged by the nurse managers, but not mandatory. Incentives were offered including continuing education units (CEU) and refreshments. The sessions were publicised by mass emailing, public address announcements, flyers posted on the inpatient units, JBVAMC Nursing Service Newsletter and telephone reminders to the charge nurses. Multiple sessions were held during all shifts, and several sessions occurred directly on the nursing units. Unit managers were kept abreast of staff attendance at these sessions via email correspondences. In addition, individual letters addressed to RNs who did not attend a session were delivered to each inpatient unit for distribution by the charge nurse or unit manager. The survey was again administered to staff on the units two months after receiving the training session. As surveys were completed on work time, staff did not receive any reimbursement for completing the surveys.

\section{Exportation of the intervention}

While in the past, the Tobacco Tactics intervention was implemented using a face-to-face, train-the-trainer model, in this study the intervention was exported via satellite broadcast from the Ann Arbor VA Medical Center (AAVAMC) to the JBVAMC. Four AAVAMC staff members were present for the 1.5 hour training of the six trainees who were present at the JBVAMC. All six trainees at the JBVAMC had some experience with conducting tobacco cessation interventions. The six trainees consisted of three nurses practitioners, one nurse educator and two pharmacists. These six trainees were responsible for disseminating the intervention in terms of training the staff nurses. AAVAMC staff also facilitated the exportation of the Tobacco Tactics intervention by mailing programme materials to the JBVAMC and training JBVAMC co-investigators regarding recruitment procedures and paperwork (via telephone conferencing). An in-person fidelity check was made by Ann Arbor personnel to the JBVAMC where three staff training sessions were observed and feedback was provided.

\section{Intervention}

\section{Patient level intervention}

The Tobacco Tactics intervention is based on an efficacious intervention previously tested (Duffy et al. 2010a) which incorporates the AHRQ recommendations for treatment of smoking and tailors the intervention to the patient's medical condition and lifestyle. The Tobacco Tactics patient toolkit includes: (1) a smoking cessation brochure (Tips for Quitting Smoking), (2) the 'Smoking: Getting Ready to Quit' video (Milner-Fenwich Inc. n.d.), (3) Tobacco Tactics manual, (4) pharmaceuticals, (5) the 1-800-QUIT-NOW help line (Smokefree.gov, http://www.smokefree.gov/expert. html; accessed 9 July 2009), and (6) follow-up telephone calls from trained veteran volunteers. Providing the brochure, video and manual in advance of cessation counselling saves the nurses' time at the bedside. The patient reviews the video on their own (shown twice daily on the overhead television at breakfast and dinner time) and meets with the staff nurses for 10-20 minutes for cessation counselling. This counselling can be broken into smaller units (e.g. four five-minute sessions) and conducted while providing routine care. A pharmaceutical protocol, initiated by the nurse and solidified by the physician, was developed. See Appendices 1 and 2 for an outline of the behavioural and pharmaceutical protocols.

\section{Nurse level intervention}

The Tobacco Tactics nurse toolkit includes: (1) one CEU for attending the Tobacco Tactics training session, (2) a PowerPoint presentation on behavioural and pharmaceutical interventions, (3) a pocket card 'Helping Smokers Quit: A 
Guide for Clinicians' (United States Department of Health and Human Services n.d.), (4) pharmaceutical and behavioural protocols, and (5) a computerised template for nurse documentation.

\section{System level interventions}

Achieving nursing buy-in. As the Tobacco Tactics programme is nurse-driven, buy-in from Nursing Service was essential. Hence, information about the Tobacco Tactics intervention was presented at several nurse-based committees such as the Nursing Education Council, the Nursing Practice Counsel and the Nursing Research Council. To secure nursing management support, information regarding the Tobacco Tactics programme was also disseminated to the nurse executives and to nurse managers during their regularly scheduled meetings.

Documentation. Documentation templates used in Ann Arbor were modified for use at the JBVAMC and activated in CPRS after approval was obtained from the JBVAMC Medical Record Committee. The template was used to document the delivery of smoking cessation services.

Tobacco Tactics materials. After securing the broadcast licensing agreement, the Patient Education Coordinator placed the 'Smoking: Getting Ready to Quit' video on the closed circuit patient education channel. The video was scheduled to play at breakfast and dinner time. The Patient Education Coordinator was identified as the appropriate person to maintain and distribute the Tobacco Tactics materials on the inpatient units. Two pharmacists who oversee the outpatient smoking cessation clinic revised the content of the Pharmaceutical Management Protocol to reflect prescribing practices at the JBVAMC.

\section{Measures}

\section{Reach and effectiveness of the programme}

To evaluate the Reach of the programme, patient survey questions asked whether or not (yes/no) they received a variety of tobacco cessation services and if they were satisfied with these services. To evaluate the Effectiveness of smoking cessation services 30 days postdischarge from the hospital, the following question was asked: 'Have you used any tobacco products in the past seven days; answer yes even if only one puff or chew of any tobacco product?' Participants were mailed a urine cotinine test kit to complete and mail back to the research team. The test kit is an inexpensive, accurate, easy-to-use method to measure a person's exposure to tobacco smoke (Studts et al. 2006). For those who refused to complete the urine test kit, we accepted a statement from the spouse or significant other regarding the patient's smoking status. Spousal proxy reports have been shown to be reliable with VA populations (Chen et al. 1995, Simon et al. 1997). For those patients who continued to smoke, harm reduction was evaluated by asking continuing smokers about the number of cigarettes/ day smoked, addiction and quit attempts. Smoking reduction is a feasible first step towards improved health and may ultimately lead to quitting in people unwilling to stop abruptly (Duffy et al. 2007, Song et al. 2008).

\section{Adoption, implementation and maintenance of the Tobacco Tactics program}

Staff survey questions rated on a five-point scale included: (1) confidence in abilities to provide tobacco cessation services, (2) perceived level of importance of providing services, (3) satisfaction with the material presented, and (4) perception of understanding the elements of the smoking cessation intervention. Nurses were also asked: (1) if they personally provided smoking cessation services to veterans (yes/no), (2) anticipated barriers to implementation (yes/no and openended), and (3) 'is there anything else the VA could do to improve the provision of smoking cessation services to veterans?' (open-ended). Results from another study using similar survey questions have been previously published (Duffy et al. 2008, 2010a).

Variables known to be associated with smoking and cessation were measured using previously validated tools. Nicotine addiction was assessed using the six-question Fagerström Test for Nicotine Dependence (FTND), a valid self-reporting measure of nicotine dependence that can assist providers in determining adequate cessation treatments (Heatherton et al. 1991). The Alcohol Use Disorders Identification Test (AUDIT) was used to measure alcohol use (Saunders et al. 1993). Depression was measured using the abbreviated Center for Epidemiologic Studies Depression Scale (CESD) (Lewinsohn et al. 1997). The Medical Outcomes Study social support survey was used to measure social support. As stress may exacerbate smoking (Todd 2004), the Perceived Stress Scale (Cohen et al. 1983) was used because it is concise and has been extensively used. Self-reported comorbidities were measured using a validated comorbidity instrument (Mukerji et al. 2007). Three belief questions, scored on a five-point Likert scale, asked about beliefs regarding the benefit to quitting. Confidence to stay off cigarettes was measured on a five-point Likert scale ranging from not at all confident to extremely confident. Age, race/ethnicity (using the US Census bureau two-tiered question), marital status, education and employment status were also collected. 


\section{Data analysis}

Mean values or frequencies were examined for all variables. Bivariate analyses compared preintervention- vs. postintervention staff and patient characteristics using Chi-square, Fisher's Exact or Student's t-tests. Chi square was used to calculate significance in categorical data (i.e. gender, employment status). As all of the respondents did not answer all of the questions, the sample size varied for different results. The Fisher's Exact test was used to calculate significance in categorical data when there was less than five respondents for a given question. Student's $t$-test was used for interval data (i.e. cigarette use, social support scale scores). Descriptive statistics (i.e. percentages) were used when reporting staff perceptions of Tobacco Tactics training as this could not be examined prior to the training sessions and hence there was no comparative data. $p$-values $<0.05$ were considered significant.

\section{Results}

\section{Reach and effectiveness of the Tobacco Tactics programme}

A total of 104 patients were recruited from October 2009 through March 2010. Table 1 shows that the baseline characteristics of patients recruited in the preintervention $(n=54)$ period compared to the postintervention period $(n=50)$ were relatively similar on all but a few characteristics. The baseline characteristics of all recruited patients are included in Table 1. Compared to postintervention patients, more preintervention patients had never married $(p=0.0456)$ and lived alone $(p=0.0271)$. Over $30 \%$ of preintervention patients and over $46 \%$ of postintervention patients $(p=0.0875)$ had problem drinking. There was also a marginal association of having greater rates of hypertension

Table 1 Baseline characteristics of smokers preintervention and postintervention $(n=104)$

\begin{tabular}{|c|c|c|c|c|c|}
\hline & \multicolumn{2}{|c|}{ Preintervention $(n=54)$} & \multicolumn{2}{|c|}{ Postintervention $(n=50)$} & \multirow[b]{2}{*}{$p$-value } \\
\hline & $n$ & Mean (SD) & $n$ & Mean (SD) & \\
\hline Number of cigarettes smoked per day in past month & 49 & $12 \cdot 67(9 \cdot 32)$ & 45 & $13 \cdot 84(13 \cdot 15)$ & $0 \cdot 6215$ \\
\hline Number of years smoked cigarettes regularly & 52 & $31 \cdot 88(13 \cdot 14)$ & 49 & $31 \cdot 30(14 \cdot 46)$ & $0 \cdot 8307$ \\
\hline Number of times quit & 42 & $4 \cdot 02(4 \cdot 49)$ & 38 & $4 \cdot 03(5 \cdot 90)$ & $0 \cdot 9983$ \\
\hline Perceived Stress Scale & 52 & $1.99(0.80)$ & 49 & $1 \cdot 81(0 \cdot 60)$ & $0 \cdot 2125$ \\
\hline Social Support Scale & $\begin{array}{l}52 \\
n(\%)\end{array}$ & $18 \cdot 54(6 \cdot 66)$ & $\begin{array}{l}49 \\
n(\%)\end{array}$ & $19 \cdot 53(6 \cdot 53)$ & $0 \cdot 4518$ \\
\hline \multicolumn{6}{|c|}{ How important do you think quitting smoking is to your health? } \\
\hline Not at all/somewhat/moderately important & \multicolumn{2}{|l|}{$9(17 \cdot 0)$} & \multicolumn{2}{|l|}{$13(26 \cdot 5)$} & \multirow[t]{2}{*}{$0 \cdot 2414$} \\
\hline Very/extremely important & \multicolumn{2}{|l|}{$44(83 \cdot 0)$} & \multicolumn{2}{|l|}{$36(73 \cdot 5)$} & \\
\hline \multicolumn{6}{|l|}{ How difficult do you think it would be to quit smoking? } \\
\hline Not at all/somewhat/moderately difficult & \multicolumn{2}{|l|}{$22(41 \cdot 5)$} & \multicolumn{2}{|l|}{$26(52 \cdot 0)$} & \multirow[t]{2}{*}{$0 \cdot 2861$} \\
\hline Very/extremely difficult & \multicolumn{2}{|l|}{$31(58 \cdot 5)$} & \multicolumn{2}{|l|}{$24(48 \cdot 0)$} & \\
\hline \multicolumn{6}{|c|}{ How likely do you think it is that quitting smoking will make you feel nervous? } \\
\hline Extremely/moderately unlikely & \multicolumn{2}{|c|}{$13(24 \cdot 5)$} & \multicolumn{2}{|l|}{$15(30 \cdot 6)$} & \multirow[t]{3}{*}{$0 \cdot 6952$} \\
\hline $50 / 50$ chance & \multicolumn{2}{|l|}{$20(37 \cdot 7)$} & \multicolumn{2}{|l|}{$19(38 \cdot 8)$} & \\
\hline Moderately/extremely likely & \multicolumn{2}{|l|}{$20(37 \cdot 7)$} & \multicolumn{2}{|l|}{$15(30 \cdot 6)$} & \\
\hline \multicolumn{6}{|c|}{ Currently thinking of quitting smoking or using other tobacco products } \\
\hline Yes, within the next 30 days & \multicolumn{2}{|l|}{$27(50 \cdot 9)$} & \multicolumn{2}{|l|}{$27(54 \cdot 0)$} & \multirow[t]{3}{*}{$0 \cdot 7513$} \\
\hline Yes, within the next six months & \multicolumn{2}{|l|}{$15(28 \cdot 3)$} & \multicolumn{2}{|l|}{$11(22 \cdot 0)$} & \\
\hline No, I am not thinking of quitting & \multicolumn{2}{|l|}{$11(20 \cdot 8)$} & $12(24 \cdot 0)$ & & \\
\hline Nicotine dependent & $24(46 \cdot 2)$ & & $20(40 \cdot 8)$ & & $0 \cdot 5887$ \\
\hline Problem drinking & & & & & \\
\hline Yes & $16(30 \cdot 2)$ & & $22(46 \cdot 8)$ & & $0 \cdot 0875$ \\
\hline No & $37(69 \cdot 8)$ & & $25(53 \cdot 2)$ & & \\
\hline Depressive symptoms & $32(61 \cdot 5)$ & & $32(64 \cdot 0)$ & & $0 \cdot 7971$ \\
\hline Self-rated health status & & & & & \\
\hline Excellent/very good & $11(21 \cdot 2)$ & & $8(16 \cdot 3)$ & & $0 \cdot 8249$ \\
\hline Good & $13(25 \cdot 0)$ & & $13(26 \cdot 5)$ & & \\
\hline Fair/poor & $28(53 \cdot 8)$ & & $28(57 \cdot 1)$ & & \\
\hline Lives alone & $26(51 \cdot 0)$ & & $14(29 \cdot 2)$ & & $0 \cdot 0271$ \\
\hline Person closest to smokes & $21(41 \cdot 2)$ & & $20(41 \cdot 7)$ & & $0 \cdot 9605$ \\
\hline Ever tried to quit smoking & $42(79 \cdot 3)$ & & $38(77 \cdot 6)$ & & $0 \cdot 8353$ \\
\hline
\end{tabular}


Table 1 (Continued)

\begin{tabular}{|c|c|c|c|c|c|}
\hline & \multicolumn{2}{|c|}{ Preintervention $(n=54)$} & \multicolumn{2}{|c|}{ Postintervention $(n=50)$} & \multirow[b]{2}{*}{$p$-value } \\
\hline & $n$ & Mean (SD) & $n$ & Mean $(\mathrm{SD})$ & \\
\hline \multicolumn{6}{|l|}{ Gender } \\
\hline Male & $52(96 \cdot 3)$ & & $50(100)$ & & \multirow[t]{2}{*}{0.4959} \\
\hline Female & $2(3 \cdot 70)$ & & $0(0 \cdot 00)$ & & \\
\hline \multicolumn{6}{|l|}{ Hispanic or Latino } \\
\hline Yes & $4(7 \cdot 41)$ & & $2(4 \cdot 00)$ & & \multirow[t]{2}{*}{0.6796} \\
\hline No & $50(92 \cdot 6)$ & & $48(96 \cdot 0)$ & & \\
\hline \multicolumn{6}{|l|}{ Race } \\
\hline White & $12(22 \cdot 2)$ & & $10(20 \cdot 0)$ & & \multirow[t]{3}{*}{0.9262} \\
\hline African American & $40(74 \cdot 1)$ & & $39(78 \cdot 0)$ & & \\
\hline Other & $2(3 \cdot 70)$ & & $1(2 \cdot 00)$ & & \\
\hline \multicolumn{6}{|l|}{ Education } \\
\hline High School or less & $15(28 \cdot 9)$ & & $22(44 \cdot 0)$ & & \multirow[t]{2}{*}{$0 \cdot 1115$} \\
\hline Some college or more & $37(71 \cdot 1)$ & & $28(56 \cdot 0)$ & & \\
\hline \multicolumn{6}{|l|}{ Marital status } \\
\hline Married & $6(11 \cdot 3)$ & & $14(28 \cdot 0)$ & & \multirow[t]{3}{*}{0.0456} \\
\hline Separated/widowed/divorced & $32(60 \cdot 4)$ & & $29(58 \cdot 0)$ & & \\
\hline Never married & $15(28 \cdot 3)$ & & $7(14 \cdot 0)$ & & \\
\hline \multicolumn{6}{|l|}{ Employment status } \\
\hline Employed & $6(11 \cdot 32)$ & & $4(8 \cdot 33)$ & & \multirow[t]{5}{*}{0.4506} \\
\hline Unemployed & $15(28 \cdot 30)$ & & $14(29 \cdot 17)$ & & \\
\hline Retired & $7(13 \cdot 21)$ & & $12(25 \cdot 00)$ & & \\
\hline Disabled & $25(47 \cdot 17)$ & & $18(37 \cdot 50)$ & & \\
\hline Homemaker & $0(0 \cdot 00)$ & & $0(0 \cdot 00)$ & & \\
\hline \multicolumn{6}{|l|}{ Medical history } \\
\hline History of cancer & $10(18 \cdot 5)$ & & $5(10 \cdot 0)$ & & $0 \cdot 2701$ \\
\hline History of lung disease & $19(35 \cdot 2)$ & & $26(52 \cdot 0)$ & & 0.0838 \\
\hline History of heart disease & $19(35 \cdot 2)$ & & $18(36 \cdot 0)$ & & 0.9309 \\
\hline History of hypertension & $36(66 \cdot 7)$ & & $41(82 \cdot 0)$ & & 0.0747 \\
\hline History of stroke & $7(13 \cdot 0)$ & & $6(12 \cdot 0)$ & & $0 \cdot 8821$ \\
\hline History of psychiatric problems & $41(75 \cdot 9)$ & & $34(68 \cdot 0)$ & & $0 \cdot 3678$ \\
\hline History of diabetes & $13(24 \cdot 1)$ & & $15(30 \cdot 0)$ & & $0 \cdot 4960$ \\
\hline History of Arthritis & $37(68 \cdot 5)$ & & $35(70 \cdot 0)$ & & $0 \cdot 8701$ \\
\hline
\end{tabular}

Bold values represent $p<0 \cdot 05$.

$(p=0.0747)$ and lung disease $(p=0.0838)$ in postintervention patients. Overall, veterans were primarily male, AfricanAmerican, single and disabled. Among both groups, probable depression rates were over $61 \%$, which is much higher than population norms of $6.7 \%$ (Kessler et al. 2005).

Table 2 shows the 30-day follow-up of veterans recruited to the preintervention group ( $n=27,50 \%$ follow-up rate) compared to the postintervention group $(n=19,38 \%$ follow-up rate). While underpowered to determine significant differences, quit rates were $3 \%$ higher in the postintervention than in the preintervention group. Smoking behaviours also changed; while the mean number of cigarettes smoked per day actually increased in the preintervention group from 13 to 15 , the mean number of cigarettes smoked per day in the postintervention group decreased from 14 to 9. Moreover, reported receipt of services was $10 \%$ higher, and satisfaction rates were also $10 \%$ higher in the postintervention patients compared to the preintervention patients. Preintervention patients were more likely to report that quitting smoking was very or extremely difficult compared to postintervention patients.

\section{Adoption, implementation and maintenance of the Tobacco Tactics programme}

A total of 31 training sessions were offered to staff at various times and in several different locations for staff convenience. Although the training sessions were open to all staff interested in smoking cessation, promotional efforts were targeted for inpatient RN staff. Of the $162 \mathrm{RNs}$ working in the inpatient units at the time of this study, over two-thirds $(67 \%, n=109)$ of the $\mathrm{RN}$ inpatient staff attended the Tobacco Tactics training over a three-month period. Table 3 
Table 2 Thirty-day follow-up to the Tobacco Tactics intervention $(n=46)$

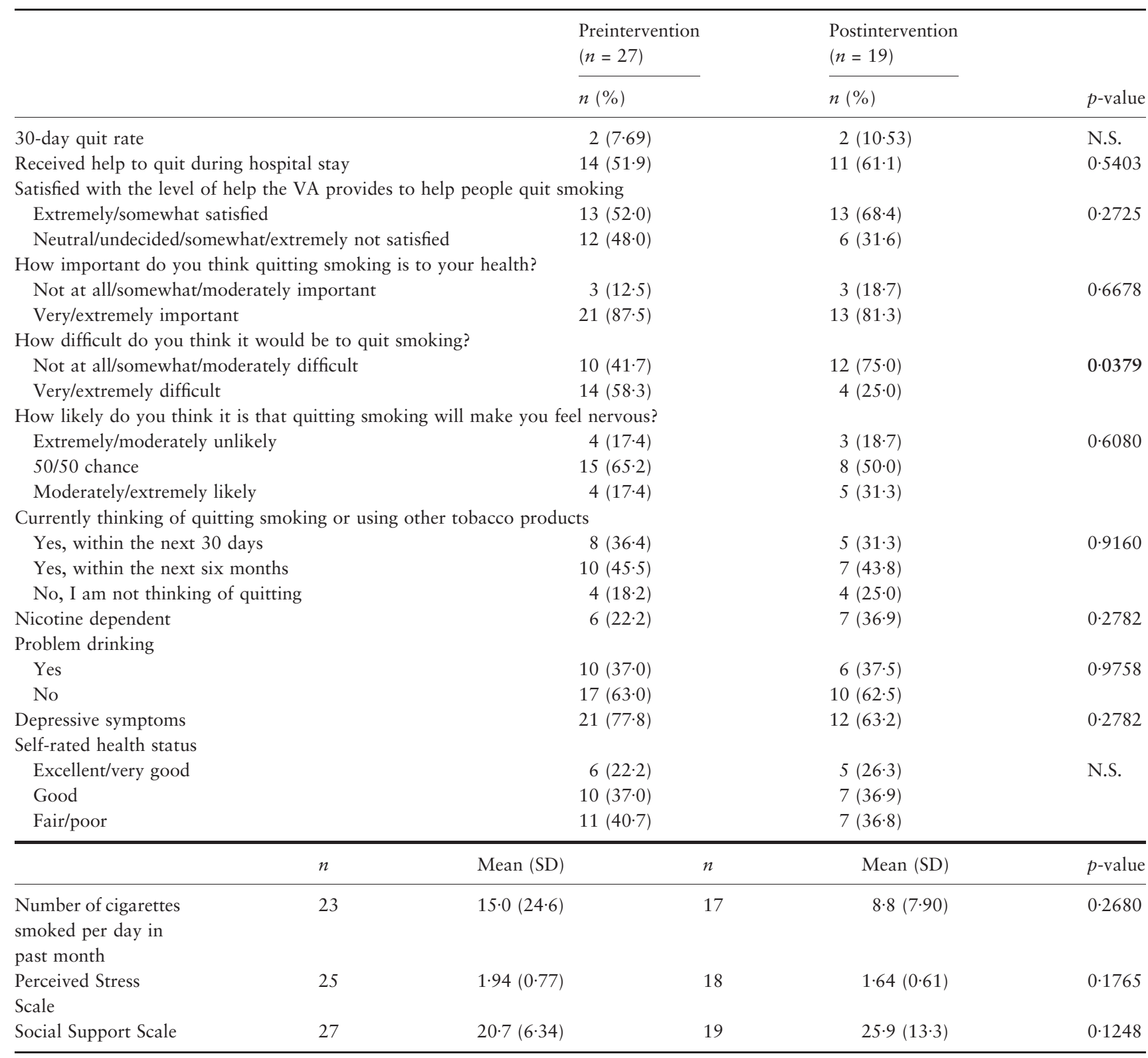

N.S., not significant with sample sizes too small to report.

Bold values represent $p<0 \cdot 05$.

shows the staff surveys from both preintervention $(n=158)$ and postintervention $(n=81)$ period. Both before and after the dissemination of the Tobacco Tactics intervention, staff felt that the VA should be doing more to assist smokers to quit and felt that providing cessation services was important. Staff confidence in their ability to provide smoking cessation services improved greatly posttraining $(p=0.0017)$ as did self-reported delivery of smoking cessation services $(p=0 \cdot 0154)$.

There were no significant demographic differences in the staff who were surveyed prior to the intervention compared to those who were surveyed after the intervention. Most of the staff were non-smokers, over age 44, woman, Asian or Black and RNs with a Bachelor of Science in Nursing (BSN) degree. Staff who did not attend the training sessions cited scheduling conflicts, competing patient care responsibilities and lacking awareness of the training schedule as barriers for attending.

Table 4 shows questions asked by staff on the two-month postintervention survey only $(n=81)$. The vast majority of staff surveyed were extremely/somewhat satisfied with the Tobacco Tactics training sessions. About two-thirds rated the behavioural and pharmaceutical management sessions as 
Table 3 Staff characteristics, attitudes and behaviours regarding cessation services preintervention and postintervention

\begin{tabular}{|c|c|c|c|}
\hline & $\begin{array}{l}\text { Preintervention } \\
(n=158)\end{array}$ & $\begin{array}{l}\text { Postintervention } \\
(n=81)\end{array}$ & \\
\hline & $n(\%)$ & $n(\%)$ & $p$-value \\
\hline $\begin{array}{l}\text { Feel the VA should be doing more to assist inpatient smokers } \\
\text { to quit smoking }\end{array}$ & $132(85 \cdot 2)$ & $65(83 \cdot 3)$ & $0 \cdot 7156$ \\
\hline \multicolumn{4}{|l|}{$\begin{array}{l}\text { How important do you think it is to provide smoking cessation } \\
\text { services to veterans? }\end{array}$} \\
\hline Not at all/somewhat/ moderately important & $16(10 \cdot 4)$ & $8(10 \cdot 1)$ & \multirow[t]{2}{*}{$0 \cdot 9501$} \\
\hline Very/extremely important & $138(89 \cdot 6)$ & $71(89.9)$ & \\
\hline \multicolumn{4}{|c|}{ How confident are you in your abilities to provide smoking cessation services to smokers? } \\
\hline Not at all/somewhat/ moderately confident & $92(60 \cdot 9)$ & $31(39 \cdot 2)$ & \multirow[t]{2}{*}{$0 \cdot 0017$} \\
\hline Very/extremely confident & $59(39 \cdot 1)$ & $48(60 \cdot 8)$ & \\
\hline Currently provide smoking cessation services to veterans & $82(54 \cdot 0)$ & $55(70 \cdot 5)$ & $0 \cdot 0154$ \\
\hline \multicolumn{4}{|l|}{ Of those who do not provide services, the reason is } \\
\hline Lack of confidence & $3(4 \cdot 29)$ & $1(4 \cdot 35)$ & N.S. \\
\hline Not enough training & $28(40 \cdot 0)$ & $6(26 \cdot 1)$ & $0 \cdot 2294$ \\
\hline Not enough time & $15(21 \cdot 4)$ & $5(21 \cdot 7)$ & N.S. \\
\hline Hesitant to upset patients & $12(17 \cdot 1)$ & $3(13 \cdot 0)$ & $0 \cdot 7547$ \\
\hline Other & $18(25 \cdot 7)$ & $7(30 \cdot 4)$ & $0 \cdot 6578$ \\
\hline \multicolumn{4}{|l|}{ Smoking status } \\
\hline Current smoker & $8(5 \cdot 16)$ & $7(8 \cdot 86)$ & \multirow[t]{3}{*}{0.5494} \\
\hline Former smoker & $24(15 \cdot 5)$ & $12(15 \cdot 2)$ & \\
\hline Never smoker & $123(79 \cdot 4)$ & $60(76 \cdot 0)$ & \\
\hline \multicolumn{4}{|l|}{ Age } \\
\hline$<35$ & $25(17 \cdot 1)$ & $8(10 \cdot 8)$ & \multirow[t]{4}{*}{$0 \cdot 3296$} \\
\hline $35-44$ & $23(15 \cdot 8)$ & $11(14 \cdot 9)$ & \\
\hline $45-54$ & $41(28 \cdot 1)$ & $29(39 \cdot 2)$ & \\
\hline $55-64 />64$ & $57(39 \cdot 0)$ & $26(35 \cdot 1)$ & \\
\hline \multicolumn{4}{|l|}{ Gender } \\
\hline Male & $24(15 \cdot 4)$ & $12(15 \cdot 8)$ & \multirow[t]{2}{*}{0.9363} \\
\hline Female & $132(84.6)$ & $64(84 \cdot 2)$ & \\
\hline \multicolumn{4}{|l|}{ Race } \\
\hline White & $20(12 \cdot 9)$ & $8(10 \cdot 4)$ & \multirow[t]{5}{*}{$0 \cdot 2861$} \\
\hline Black & $44(28.4)$ & $19(24 \cdot 7)$ & \\
\hline Hispanic/Latino & $5(3 \cdot 23)$ & $3(3 \cdot 90)$ & \\
\hline Asian/Pacific Islander & $82(52.9)$ & $40(52 \cdot 0)$ & \\
\hline American Indian/ Eskimo/Aleutian/ other & $4(2 \cdot 58)$ & $7(9 \cdot 09)$ & \\
\hline \multicolumn{4}{|l|}{ Four-year college degree } \\
\hline Yes & $126(80 \cdot 3)$ & $59(76 \cdot 6)$ & \multirow[t]{2}{*}{$0 \cdot 5212$} \\
\hline No & $31(19 \cdot 7)$ & $18(23 \cdot 4)$ & \\
\hline \multicolumn{4}{|l|}{ Current position } \\
\hline $\mathrm{RN}$ & $120(78 \cdot 4)$ & $63(81 \cdot 8)$ & \multirow[t]{3}{*}{$0 \cdot 8157$} \\
\hline LPN/nursing assistant & $13(8 \cdot 50)$ & $6(7 \cdot 80)$ & \\
\hline Other professional & $20(13 \cdot 1)$ & $8(10 \cdot 4)$ & \\
\hline
\end{tabular}

N.S., not significant with sample sizes too small to report.

Bold values represent $p<0 \cdot 05$.

excellent/very good. Over 92\% strongly agreed or agreed that they had a good understanding of the elements of the Tobacco Tactics intervention and about $85 \%$ felt that the intervention would be helpful to veterans. The most commonly provided services were advice, counselling, handout materials and medications. On average, staff spent about 11.6 minutes counselling the average smoker. Unfortunately, postdischarge telephone calls were not initiated, as it was difficult to identify staff to conduct the follow-up process. 
Table 4. Staff perceptions about and responses to Tobacco Tactics training $(n=101)$

\begin{tabular}{|c|c|c|}
\hline & $n$ & $\%$ \\
\hline $\begin{array}{l}\text { Participated in training for smoking } \\
\text { cessation }\end{array}$ & 81 & $80 \cdot 2$ \\
\hline \multicolumn{3}{|l|}{ Satisfied with the material presented } \\
\hline Extremely/somewhat satisfied & 71 & $87 \cdot 7$ \\
\hline $\begin{array}{l}\text { Neutral/undecided/somewhat/ } \\
\text { extremely not satisfied }\end{array}$ & 10 & $12 \cdot 3$ \\
\hline \multicolumn{3}{|l|}{ Pharmaceutical management session } \\
\hline Excellent/very good & 51 & $69 \cdot 9$ \\
\hline Good/fair/poor & 22 & $30 \cdot 1$ \\
\hline \multicolumn{3}{|l|}{ Behavioural management session } \\
\hline Excellent/very good & 23 & $69 \cdot 7$ \\
\hline Good/fair/poor & 10 & $30 \cdot 3$ \\
\hline \multicolumn{3}{|c|}{$\begin{array}{l}\text { Good understanding of the elements of the Smoking Cessation } \\
\text { Intervention }\end{array}$} \\
\hline Strongly agree/agree & 75 & $92 \cdot 6$ \\
\hline Neutral/disagree/strongly disagree & 6 & $7 \cdot 40$ \\
\hline \multicolumn{3}{|c|}{$\begin{array}{l}\text { How helpful do you think the smoking cessation programme you } \\
\text { received training on is to veteran smokers? }\end{array}$} \\
\hline Extremely/somewhat helpful & 67 & $84 \cdot 8$ \\
\hline $\begin{array}{l}\text { Neutral/undecided/somewhat/ } \\
\text { extremely unhelpful }\end{array}$ & 12 & $15 \cdot 2$ \\
\hline \multicolumn{3}{|c|}{ Of those who do provide services, which do they provide? } \\
\hline Advice $(n=55)$ & 53 & $96 \cdot 4$ \\
\hline Individual counselling $(n=36)$ & 26 & $72 \cdot 2$ \\
\hline Group counselling $(n=46)$ & 20 & $43 \cdot 5$ \\
\hline Medications $(n=33)$ & 23 & $70 \cdot 0$ \\
\hline Hand-out materials $(n=55)$ & 50 & $91 \cdot 0$ \\
\hline Video about quitting smoking $(n=34)$ & 11 & $32 \cdot 4$ \\
\hline \multirow[t]{2}{*}{ Phone calls from the VA $(n=43)$} & 15 & $35 \cdot 0$ \\
\hline & Mean & SD \\
\hline $\begin{array}{l}\text { Average number of minutes spent } \\
\text { counselling the average smoker } \\
(\min =0 ; \max =37.5)\end{array}$ & $11 \cdot 60$ & $9 \cdot 50$ \\
\hline
\end{tabular}

\section{Discussion}

There was an improvement in the Reach and Effectiveness of the Tobacco Tactics intervention postintervention as compared to preintervention. In terms of Reach, veteran smokers reported an increase in receipt of cessation services and satisfaction with these services postintervention compared to preintervention. This finding is congruent with a Cochrane review which demonstrated that health professional training increases the delivery of smoking cessation interventions (Lancaster et al. 2000). In terms of Effectiveness, albeit nonsignificant, there was a modest improvement in 30-day quit rates in the postintervention group compared to the preintervention group. Research has shown that when smoking cessation services are offered, quit rates increase (Doll et al. 2004).

Moreover, for those still smoking, there was also a substantial decrease in the number of cigarettes smoked per day. Although not as advantageous as smoking cessation, reduction in tobacco consumption results in numerous health benefits. In a systematic review, tobacco use reduction was found to decrease morbidity by reducing the incidence of smoking-related cancers, improve respiratory symptoms and decrease the risk of cardiovascular disease (Pisinger \& Godtfredsen 2007). In addition, lowering cigarette consumption improves cessation rates (Song et al. 2008).

There were some differences in the characteristics between the preintervention and postintervention groups that may have affected quit rates. Both groups screened over three times higher than population norms for problem drinking which can be largely attributed to the fact that $50 \%$ of patients recruited were admitted to the psychiatric unit. Compared to the preintervention group, most of those recruited to the postintervention group screened positive for problem drinking; yet at 30-day follow-up this difference was no longer present suggesting that many of the problem drinkers were perhaps non-responders accounting for the lower response rate in this group. Problem drinking, common among veterans (Lambert et al. 2005) is associated with smoking, higher nicotine dependence and greater difficulty quitting (Dawson 2000, Leeman et al. 2008).

While there were no differences between the groups in probable depression rates, depression rates were alarmingly six times the population norms. The disproportionate number of psychiatric patients included in this sample may explain the high depression rate. Patients hospitalised primarily for psychiatric disorders may be just as motivated to quit smoking than inpatients admitted for medical reasons (Siru et al. 2010). However, depression is highly correlated with smoking, and depressed smokers often have a harder time quitting; the high rates of depression coupled with the high rates of drinking among this sample make smoking cessation particularly challenging (Duffy et al. 2002, 2006, 2010b, Lambert et al. 2005). Combination interventions that target multiple behaviours/conditions (i.e. smoking, alcohol and depression) such as the one implemented by Duffy et al. (2006), may be particularly helpful for veteran smokers with multiple behavioural and psychological comorbidities. At the 2009 National Institutes of Health (NIH) meeting on the Science of Behavior Change (NIH 2009), it was acknowledged that risk behaviours often occur in 'bundles' and we should move away from focusing on one disorder at a time.

There were more smokers with hypertension and lung disease in the postintervention group. Comorbidities have been shown to be associated with motivation to quit smoking. For example, smokers with lung disease tend to be more nicotine dependent and have a decreased readiness to quit (Jimenez-Ruiz et al. 2001). In addition, patients with 
lung disease have a higher incidence of depression that could adversely influence their willingness to quit smoking (Wagena et al. 2005).

Smokers in the preintervention group were more likely than those in the postintervention group to believe that quitting smoking would be difficult at 30-day follow-up. Perceived difficult quitting is a marker of self-efficacy and self-efficacy to quit smoking has had mixed results in the literature. While some studies show that perceived high selfefficacy can enhance quitting, others show that those with perceived high self-efficacy are less likely to perceive the need to participate in interventions (Duffy et al. 2010b).

In terms of the Adoption, Implementation and Maintenance phases of the RE-AIM framework, this study showed that, once packaged into a toolkit, the nurse-administered Tobacco Tactics intervention can be easily transported to another institution. By mailing the components of the toolkit to the institution and training six trainers via satellite broadcast, over two-thirds of the nurses were trained during a three-month period. Overall, staff were very satisfied with the training sessions, confidence levels improved and consequently the provision of services improved.

Despite the success of transporting the Tobacco Tactics intervention to the JBVAMC, there were several barriers to implementation. Although attendance was strongly encouraged by unit managers among nursing personnel, some nurses were not relieved of patient care responsibilities to attend the Tobacco Tactics training. Several staff members accepted the role of Tobacco Tactics trainer; however, discomfort in presenting course materials and competing demands limited their availability.

Planning is ongoing to improve the Effectiveness, Adoption and Maintenance of the Tobacco Tactics intervention at the JBVAMC. Relaxing prescribing practices and launching postdischarge follow-up calls are two strategies that will be pursued to enhance the Effectiveness of the Tobacco Tactics intervention. To ensure sustainability of the intervention, in collaboration with Nursing Education, the Tobacco Tactics training will be incorporated into nursing orientation and will also be offered online via the Mosby's Nursing Skills website for annual review by the nursing staff. Lastly, medical residents will be offered brief training during regularly scheduled conferences.

\section{Limitations}

This study was a preintervention and postintervention study without a comparison group and was therefore unable to control historical effects that may influence the results. While a predesign postdesign is not as strong as other designs that offer comparison groups, implementation research, which is designed to get evidence into practice, often uses predesign postdesigns because of the low cost, convenience and simplicity (Songer n.d.). Although the 30-day quit rates were biochemically verified, 30-day quit rates are not as reliable as six-month quit rates, but have been found to be highly correlated with six-month quit rates and are thus a reasonable marker to measure smoking for short-term evaluation projects (Ockene et al. 2000). While the inclusion of selfreported questionnaires and return of urine cotinine samples are standard measures in tobacco studies (Studts et al. 2006), both the preintervention and postintervention response rates were low perhaps because of factors such as problem drinking, low SES, unstable housing and/or the possibility that these hospitalised smokers became sicker and were unable to respond to the survey. Although patients were referred to the 1-800-QUIT-NOW telephone hotline for follow-up counselling, proactive telephone outreach from the VA was not yet implemented which is problematic as postdischarge cessation telephone support has been shown to improve quit rates (Chouinard \& Robichaud-Ekstrand 2005, Davies et al. 2005, Stansby \& McCaslin 2006, Gies et al. 2008, Duffy et al. 2010a, Siru et al. 2010).

\section{Relevance to clinical practice}

In July 2011, the Joint Commission released new standards for inpatient smoking, which include screening for tobacco use, provision of treatment during the hospital stay, provision of treatment at discharge and one month postdischarge follow-up; the nurse-delivered Tobacco Tactics intervention meets these standards. Using the RE-AIM implementation framework, this implementation study showed that the Tobacco Tactics intervention can be easily transported to other hospitals. Evaluation of the Reach of the programme showed that smokers reported greater receipt of services and were more satisfied with services postintervention. Evaluation of the Effectiveness of the programme showed that quit rates improved, and for those smoking, the number of cigarettes decreased in the postintervention group. In terms of the Adoption, Implementation and Maintenance of the Tobacco Tactics programme, nurses were satisfied with the training, showed improved confidence after the training, increased their delivery of cessation services after the training and continued to provide cessation services. Hence, the Tobacco Tactics intervention has the potential to be widely disseminated and is currently being tested in a large NIH-funded study outside of the VA system. Wide-scale dissemination of the nurse-delivered Tobacco Tactics intervention has the potential to reduce smoking-related morbidity and mortality. 


\section{Acknowledgements}

First and foremost, the authors would like to express our heartfelt appreciation to the Jesse Brown nurses who included the intervention in their already busy work schedules. We would also like to thank the veterans who participated in this study. Last, we would like to thank the Department of Veterans Affairs for their generous support of this Service Directed Project (SDP 06-003).

\section{Contributions}

Study design: LV, SD, KR; data collection and analysis: LV, LE, KR and manuscript preparation: LV, SD, LE, KR, CZ.

\section{Conflict of interest}

No conflicts of interest to declare.

\section{References}

Abildso CG, Zizzi SJ \& Reger-Nash B (2010) Evaluating an insurancesponsored weight management program with the RE-AIM Model, West Virginia, 2004-2008. Preventing Chronic Disease 7, A46.

Anesetti-Rothermel A, Noerachmanto N, Horn K \& Dino G (2011) Beyond reach and effectiveness: evaluating the NotOn-Tobacco (N-O-T) Program in West Virginia from 2000 to 2005. Health Promotion Practice, In press.

Bakken S \& Ruland CM (2009) Translating clinical informatics interventions into routine clinical care: how can the REAIM framework help? Journal of the American Medical Informatics Association 16, 889-897.

Caperchione C \& Coulson F (2010) The WellingTONNE challenge toolkit: using the RE-AIM framework to evaluate a community resource promoting healthy lifestyle behaviors. Health Education Journal 69, 126-134.

Centers for Disease Control and Prevention (2007) Best Practices for Comprehensive Tobacco Control Programs. CDC, Atlanta, GA.

Chen Y, Rennie DC \& Dosman JA (1995) The reliability of cigarette consumption reports by spousal proxies. American Journal of Public Health 85, 17111712.

Chouinard MC \& Robichaud-Ekstrand S (2005) The effectiveness of a nursing inpatient smoking cessation program in individuals with cardiovascular disease. Nursing Research 54, 243-254.

Cohen S, Kamarck $\mathrm{T} \&$ Mermelstein R (1983) A global measure of perceived stress. Journal of Health and Social Behavior 24, 385-396.

Cui Y, Wen W, Moriarty CJ \& Levine RS (2006) Risk factors and their effects on the dynamic process of smoking relapse among veteran smokers. Behavior Resesearch and Therapy 44, 967-981.

Dall TM, Zhang Y, Chen YJ, Wagner RC, Hogan PF, Fagan NK, Olaiya ST \& Tornberg DN (2007) Cost associated with being overweight and with obesity, high alcohol consumption, and tobacco use within the military health system's TRICARE prime-enrolled population. American Journal of Health Promotion 22, 120-139.

DaviesSL,KohlerCL,FishL, Taylor BE,Foster GE \& Annang L (2005) Evaluation of an intervention for hospitalized African American smokers. American Journal of Health Behavior 29, 228-239.

Dawson DA (2000) Drinking as a risk factor for sustained smoking. Drug and Alcohol Dependence 59, 235-249.

Dillman DA (1978) Mail and Telephone Surveys: The Total Design Method. John Wiley \& Sons, New York, NY.

Doll R, Peto R, Boreham J \& Sutherland I (2004) Mortality in relation to smoking: 50 years' observations on male British doctors. British Medical Journal 328, 1519.

Duffy SA, Terrell JE, Valenstein M, Ronis DL, Copeland LA \& Connors M (2002) Effect of smoking, alcohol, and depression on the quality of life of head and neck cancer patients. General Hospital Psychiatry 24, 140-147.

Duffy SA, Ronis DL, Valenstein M, Lambert MT, Fowler KE, Gregory L, Bishop C, Myers LL, Blow FC \& Terrell JE (2006) A tailored smoking, alcohol, and depression intervention for head and neck cancer patients. Cancer Epidemiology, Biomarkers \& Prevention 15, 2203-2208.

Duffy SA, Ronis DL, Valenstein M, Fowler KE, Lambert MT, Bishop C \& Terrell
JE (2007) Depressive symptoms, smoking, drinking, and quality of life among head and neck cancer patients. Psychosomatics 48, 142-148.

Duffy SA, Reeves P, Hermann C, Karvonen C \& Smith P (2008) In-hospital smoking cessation programs: what do VA patients and staff want and need? Applied Nursing Research 21, 199-206.

Duffy SA, Karvonen-Gutierrez CA, Ewing LA, Smith PM \& Veterans Integrated Services Network 11 Tobacco Tactics Team (2010a) Implementation of the Tobacco Tactics program in the Department of Veterans Affairs. Journal of General Internal Medicine 25, 3-10.

Duffy SA, Scheumann A, Fowler KE, Darling-Fisher C \& Terrell JE (2010b) Perceived difficulty quitting predicts enrollment in a smoking-cessation program for patients with head and neck cancer. Oncology Nursing Forum 37, 349-356.

Ergul S \& Temel AB (2009) The effects of a nursing smoking cessation intervention on military students in Turkey. International Nursing Review 56, 102-108.

Fiore MC, Jaen CR, Baker TB, Bailey WC, Benowitz NL, Curry SJ, Dorfman SF, Froelicher ES, Goldstein MG, Healton CG, Henderson PN, Heyman RB, Koh HK, Kottke TE, Lando HA, Mecklenburg RE, Mermelstein RJ, Mullen PD, Orleans CT, Robinson L, Stitzer ML, Tommasello AC, Villejo L \& Wewers ME (2008) Treating Tobacco Use and Dependence: 2008 Update: Clinical Practice Guideline. U.S. Department of Health and Human Services, Public Health Service, Rockville, MD.

France EK, Glasgow RE \& Marcus AC (2001) Smoking cessation interventions among hospitalized patients: what have 
we learned? Preventive Medicine 32, 376-388.

Gies CE, Buchman D, Robinson J \& Smolen D (2008) Effect of an inpatient nursedirected smoking cessation program. Western Journal of Nursing Research 30, 6-19.

Ginn MB, Cox G \& Heath J (2008) Evidence-based approach to an inpatient tobacco cessation protocol. AACN Advanced Critical Care 19, 268-278, quiz 279-280.

Glasgow RE, Klesges LM, Dzewaltowski DA, Estabrooks PA \& Vogt TM (2006) Evaluating the impact of health promotion programs: using the RE-AIM framework to form summary measures for decision making involving complex issues. Health Education Research 21, 688-694.

Gomm M, Lincoln P, Egeland P \& Rosenberg M (2002) Helping hospitalised clients quit smoking: a study of rural nursing practice and barriers. Australian Journal of Rural Health 10, 26-32.

Hamlett-Berry K, Davison J, Kivlahan DR, Matthews MH, Hendrickson JE \& Almenoff PL (2009) Evidence-based national initiatives to address tobacco use as a public health priority in the Veterans Health Administration. Military Medicine 174, 29-34.

Heatherton TF, Kozlowski LT, Frecker RC \& Fagerstom KO (1991) The Fagerstrom Test for Nicotine Dependence: a revision of the Fagerstrom Tolerance Questionnaire. British Journal of Addiction 86, 1119-1127.

Hennrikus DJ, Lando HA, McCarty MC, Klevan D, Holtan N, Huebsch JA, Jestus S, Pentel PR, Pine D, Sullivan S, Swenson K \& Vessey J (2005) The TEAM project: the effectiveness of smoking cessation intervention with hospital patients. Preventive Medicine 40, 249-258.

Jimenez-Ruiz CA, Masa F, Miravitlles M, Gabriel R, Viejo JL, Villasante C \& Sobradillo V (2001) Smoking characteristics: differences in attitudes and dependence between healthy smokers and smokers with COPD. Chest 119, 1365-1370.

Kaiser Permanente Colorado Region Institute for Health Research (2010) RE-AIM. Available at: http://www. re-aim.org/ (accessed 7 July 2010).
Kessler R, Chiu W, Demler O, Merikangas KR \& Walters EE (2005) Prevalence, severity, and comorbidity of twelvemonth DSM-IV disorders in the National Comorbidity Survey Replication (NCS-R). Archives of General Psychiatry 62, 617-627.

Lambert M, Terrell JE, Copeland LA, Ronis DL \& Duffy SA (2005) Cigarettes, alcohol, and depression: characterizing head and neck cancer survivors in two systems of care. Nicotine \& Tobacco Research 7, 233-241.

Lancaster T, Silagy C \& Fowler G (2000) Training health professionals in smoking cessation. Cochrane Database of Systematic Reviews, Issue 3, Art. No.: CD000214.

Leeman RF, McKee SA, Toll BA, KrishnanSarin S, Cooney JL, Makuch RW \& O'Malley SS (2008) Risk factors for treatment failure in smokers: relationship to alcohol use and to lifetime history of an alcohol use disorder. Nicotine \& Tobacco Research 10, 1793-1809.

Lewinsohn PM, Seeley JR, Roberts RE \& Allen NB (1997) Center for Epidemiologic Studies Depression Scale (CESD) as a screening instrument for depression among community-residing older adults. Psychology and Aging 12, 277-287.

Meyer C, Ulbricht S, Gross B, Kastel L, Wittrien S, Klein G, Skoeries BA, Rumpf HJ \& John U (2011) Adoption, reach and effectiveness of computerbased, practitioner delivered and combined smoking interventions in general medical practices: a three-arm cluster randomized trial. Drug and Alcohol Dependence 121, 124-132.

Miller NH, Smith PM, DeBusk RF, Sobel DS \& Taylor CB (1997) Smoking cessation in hospitalized patients. Results of a randomized trial. Archives of Internal Medicine 157, 409-415.

Milner-Fenwich Inc. (n.d.) Smoking: Getting Ready to Quit, 2nd edn. Available at: http://www.milnder-fenwick.com/ products/gn51/index.asp\#details (accessed 9 July 2009).

Mukerji SS, Duffy SA, Fowler KE, Khan M, Ronis DL \& Terrell JE (2007) Comorbidities in head and neck cancer: agreement between self-report and chart review. Otolaryngology, Head and Neck Surgery 136, 536-542.
National Institutes of Health (2009) Meeting Summary, NIH Science of Behavior Change. National Institutes of Health, Bethesda, MD.

Ockene JK, Emmons KM, Mermelstein RJ, Perkins KA, Bonollo DS, Voorhees CC \& Hollis JF (2000) Relapse and maintenance issues for smoking cessation. Health Psychology 19, 17-31.

Pisinger C \& Godtfredsen NS (2007) Is there a health benefit of reduced tobacco consumption? A systematic review. Nicotine \& Tobacco Research 9, 631-646.

Prochaska JJ, Fromont SC, Hudmon KS \& Cataldo JK (2009) Designing for dissemination: development of an evidence-based tobacco treatment curriculum for psychiatry training programs. Journal of American Psychiatric Nurses Association 15, 24-31.

Reid RD, Mullen KA, Slovinec D'Angelo ME, Aitken DA, Papadakis S, Haley PM, McLaughlin CA \& Pipe AL (2010) Smoking cessatin for hospitalized smokers: an evaluation of the "Ottawa Model." Nicotine Tobacco Research $12,11-18$.

Rice VH \& Stead LF (2008) Nursing interventions for smoking cessation. Cochrane Database of Systematic Reviews, Issue 1, Art. No.: CD001188.

Rigotti N, Munafo MR \& Stead LF (2007) Interventions for smoking cessation in hospitalised patients. Cochrane Database of Systematic Reviews, Issue 5, Art. No.: CD001837.

Saunders JB, Aasland OG, Babor TF, de la Fuente JR, Grant M (1993) Development of the Alcohol Use Disorders Identification Test (AUDIT): WHO collaborative project on early detection of persons with harmful alcohol consumptionII. Addiction 88, 791804.

Sherman SE, Joseph AM, Yano EM, Simon BF, Arikian N, Rubenstein LV, Parkerton P \& Mittman BS (2006) Assessing the institutional approach to implementing smoking cessation practice guidelines in veterans health administration facilities. Military Medicine 171, 80-87.

Sherman SE, Takahashi N, Kalra P, Gifford E, Finney JW, Canfield J, Kelly JF, Joseph GJ \& Kuschner W (2008) Care coordination to increase referrals to smoking cessation telephone 
counseling: a demonstration project. American Journal of Managed Care 14, 141-148.

Simon JA, Solkowitz SN, Carmody TP \& Browner WS (1997) Smoking cessation after surgery. A randomized trial. Archives of Internal Medicine 157, 1371-1376.

Siru R, Hulse GK, Khan RJK \& Tait RJ (2010) Motivation to quit smoking among hospitalised individuals with and without mental health disorders. Australian and New Zealand Journal of Psychiatry 44, 640-647.

Song YM, Sung J \& Cho HJ (2008) Reduction and cessation of cigarette smoking and risk of cancer: a cohort study of Korean men. Journal of Clinical Oncology 26, 5101-5106.

Songer T (n.d.). Before and After Studies in Injury Research. University of Pittsburgh, Pittsburgh, PA.

Stansby G \& McCaslin J (2006) Targeting smokers in the hospital setting. British

\section{Appendix 1}

\section{Smoking cessation behavioural management protocol}

Assess if patient interested in quitting

If patient not interested, leave brochure at bedside

If patient interested, leave brochure and arrange for patient to view video

After video, provide patient with patient manual to read if able

Using patient manual, assist patient with behavioural intervention including

Self assessment

Smoker type

Smoking costs

Handling cravings

Relapse prevention

Medication options

Along with patient, identify and arrange for cessation medications (see pharmaceutical protocol)

Arrange for follow-up calls
Journal of Hospital Medicine 67, 138140.

tudts JL, Ghate SR, Gill JL, Studts CR, Barnes CN, LaJoie AS, Andrykowski MA \& LaRocca RV (2006) Validity of self-reported smoking status among participants in a lung cancer screening trial. Cancer Epidemiology Biomarkers \& Prevention 15, 1825-1828.

Targhetta R, Bernhard L, Sorokaty J-M, Balmes J-L, Nalpas B \& Perney P (2011) Intervention study to improve smoking cessation during hospitalization. Public Health 125, 457-463.

The Center for Nursing Advocacy (2006) How Many Nurses are There? And Other Facts. Available at: http://www. nursingadvocacy.org/faq/rn_facts.html (accessed 6 June 2009). cquit rate results and views of participants. Health Education Journal 66, 307-322.
Titler MG (2004) Methods in translation science. Worldviews on Evidence-Based Nursing 1, 38-48.

Todd M (2004) Daily processes in stress and smoking: effects of negative events, nicotine dependence, and gender. Psychology of Addictive Behaviors 18, 31-39.

U.S. Department of Health and Human Services (n.d.) Helping Smokers Quit: A Guide for Clinicians. Available at: http://www.ahrq.gov/clinic/tobacco/clin hlpsmksqt.pdf (accessed 9 July 2009).

Wagena EJ, Arrindell WA, Wouters EF \& van Schayck CP (2005) Are patients with COPD psychologically distressed? European Respiratory Journal 26, 242248.

Wolfenden L, Campbell E, Wiggers J, Walsh RA \& Bailey LJ (2008) Helping hospital patients quit: what the evidence supports and what guidelines recommend. Preventive Medicine 46, 346-357.

\section{Appendix 2}

\section{Smoking cessation pharmaceutical management protocol}

Recommend nicotine replacement (patch, gum or lozenge) if Never used patch, gum or lozenge before

Used patch, gum or lozenge successfully in the past (smoke-free greater than three months)

Recommend nicotine replacement (patch AND gum OR

lozenge) if

Smoke greater than one pack per day

Failed nicotine replacement therapy in past

Recommend referral to appropriate service (i.e. pulmonary, psychiatry and/or pharmacy/addiction therapy clinic) for a thorough evaluation if

Failed nicotine replacement therapy in the past (smoke-free less than three months)

Failed nicotine replacement and bupropion monotherapy in the past

Patch, gum or lozenge intolerant (i.e. rash, etc.)

History of depression or currently has depressive symptoms

Intolerance or treatment failure to nicotine replacement and bupropion 
The Journal of Clinical Nursing (JCN) is an international, peer reviewed journal that aims to promote a high standard of clinically related scholarship which supports the practice and discipline of nursing.

For further information and full author guidelines, please visit JCN on the Wiley Online Library website: http:// wileyonlinelibrary.com/journal/jocn

\section{Reasons to submit your paper to JCN:}

High-impact forum: one of the world's most cited nursing journals, with an impact factor of 1·118 - ranked 30/95 (Nursing (Social Science)) and 34/97 Nursing (Science) in the 2011 Journal Citation Reports ${ }^{\circledR}$ (Thomson Reuters, 2011)

One of the most read nursing journals in the world: over 1.9 million full text accesses in 2011 and accessible in over 8000 libraries worldwide (including over 3500 in developing countries with free or low cost access).

Early View: fully citable online publication ahead of inclusion in an issue.

Fast and easy online submission: online submission at http://mc.manuscriptcentral.com/jcnur.

Positive publishing experience: rapid double-blind peer review with constructive feedback.

Online Open: the option to make your article freely and openly accessible to non-subscribers upon publication in Wiley Online Library, as well as the option to deposit the article in your preferred archive. 